\title{
Debates intersexuales entre Colombia y España. Sobre las teorías científicas del médico español Gregorio Marañón y Posadillo (1887-1960) y la normativización de los cuerpos intersexuales en Colombia en la primera mitad del siglo $\mathrm{XX}^{*}$.
}

\author{
Sara Lugo-Márquez ${ }^{* *}$
}

Universitat Autònoma de Barcelona

\section{Resumen}

La intersexualidad ha sido para la ciencia positiva una paradoja por transgredir las categorías de sexo y género en las cuales se pretenden encajar los cuerpos. Para perpetuar dichas categorías la medicina ha desarrollado un complejo sistema de patologización que le permite el control de los cuerpos intersexuados. Asimismo, la separación entre género y sexo ha sido recientemente cuestionada por los análisis feministas de las ciencias y las tecnologías al plantearse que el sexo es un resultado del género, ya que los discursos biológicos son construcciones sociales. Ambas perspectivas permiten entender la patologización de la intersexualidad como causa y consecuencia de los procesos de medicalización de la infancia y de la sexualidad que se consolidaron a principios del siglo XX. El presente artículo evidencia la circulación de las teorías científicas mundialmente difundidas del médico español Gregorio Marañón y Posadillo (1887-1960) sobre «los estados intersexuales» en Colombia en la primera mitad del siglo XX, y como éstas fueron apropiadas y transformadas por el endocrinólogo colombiano Francisco Gnecco Mozo (1904-1973), quien fue estudiante en España de Gregorio Marañón y perpetuador del estudio de la intersexualidad en Colombia. Además, se plantean dos contribuciones epistemológicas de los médicos colombianos para reafirmar la heteronormatividad y la

\footnotetext{
* Este artículo corresponde a resultados preliminares en el marco del proyecto de investigación: Postcolonial genealogy of intersexuality. Influence of US and Spanish scientific theories in the Colombian medical practices and discourses about intersexuality in the second half of twentieth century, el cual ha recibido financiación del programa European Union's Horizon 2020 Research and Innovation - Marie SklodowskaCurie, grant agreement No 703966.

** Bióloga, MA y PhD en Historia de la Ciencia por la Universitat Autònoma de Barcelona. Actualmente investigadora Postdoctoral, Marie S. Curie (IF) en el Centre d'Història de la Ciència (CEHIC) - Unitat d'Història de la Medicina (Facultat de Medicina) - Universitat Autònoma de Barcelona. Email: sara.lugo@uab.cat.
} 
construcción de una normativa de los cuerpos, que esperamos tracen un análisis preliminar de la patologización de la intersexualidad en el discurso médico colombiano de la primera mitad del siglo XX.

\title{
Palabras Claves
}

Intersexualidad; patologización; heteronormatividad; género, ciencia y tecnología, historia de la medicina; medicalización.

\begin{abstract}
Intersexuality has been a paradox for the positive science because it transgresses the sex and gender categories in which they pretend to fit bodies in. To perpetuate these categories medicine has developed a complex system of pathologization that allows the control of intersexed bodies. Likewise, the separation between sex and gender has been recently questioned by feminist analysis of sciences and technologies, when considering that sex is a gender result, since biological discourses are social constructions. Both perspectives permit the understanding of intersex pathologization as a cause and consequence of childhood and sexuality medicalization processes, which had been consolidated in the beginning of the 20th century. This paper evidences the worldwide spreading of scientific theories developed by the Spanish physician Gregorio Marañón y Posadillo (1887-1960) about «los estados intersexuales» in Colombia in the first half of the 20th century, and how these were appropriated and transformed by the Colombian endocrinologist Francisco Gnecco Mozo (1904-1973), who was Gregorio Marañón's student in Spain and a promoter of the study of intersex in Colombia. In addition, we suggest two epistemological contributions of Colombian physicians to reaffirm heteronormativity and the construction of a bodies normative that we hope would sketch a preliminary analysis of intersex pathologization in the Colombian medical discourses in the first half of the 20th century.
\end{abstract}

\section{Key words}


Intersex; pathologization; heteronormativity; gender, science and technology; history of medicine.

\section{La intersexualidad, ¿en qué estamos? del cuerpo orgánico al cuerpo social y de nuevo al cuerpo orgánico}

Desde la invención de la ciencia positiva, la intersexualidad se ha convertido en paradoja de transgresión a los parámetros de normalidad impuestos por el proceso de medicalización. Consecuentemente, la medicina positiva construyó una estructura compleja de patologización para encajar a las personas intersexuales, y en general a todos los individuos, en las categorías normativas de hombre y mujer. Desde 1990, con los estudios de Suzanne J. Kessler sobre La construcción médica del género: el manejo de casos de infantes intersexuales, se abrió la discusión sobre los argumentos médicos para el tratamiento quirúrgico y hormonal de infantes intersexuales, centrando la problemática en si se tienen en cuenta los riesgos y la calidad de vida de la persona intersexual medicalizada, o si por el contrario a quien se está protegiendo es a la «cultura del infante» (Kessler, 1990). Las discusiones que plantean los análisis sobre la intersexualidad proponen discusiones no sólo históricas sobre la construcción de procesos de patologización, sino también epistemológicas y filosóficas que nutren, y se nutren del pensamiento feminista y queer contemporáneos.

Histórica y políticamente entendemos la necesidad de la demarcación entre género y sexo realizada por el feminismo de la «segunda ola» ${ }^{1}$. Sin embargo, esto condujo a la no problematización del discurso científico referente al cuerpo, al abandono de la diversidad orgánica y a la radicalización de teorías como la de Jhon Money $\left(1921\right.$ - 2006) ${ }^{2}$, que

\footnotetext{
${ }^{1}$ Utilizamos comillas para referirnos a la periodización tradicional de los momentos históricos del feminismo porque consideramos que no es posible realizar tal demarcación. Por ejemplo, no es adecuado, y además es eurocéntrico, afirmar que el "periodo sufragista» haya terminado, ya que es una lucha actual de diversos contextos geopolíticos.

2 Académicamente, John Money ha sido reconocido como una de las personas más influyentes en la sexología de la segunda mitad del siglo XX (Bullough, 2003). Algunas investigaciones plantean que fue quien conceptualizó el término género como la dimensión social y cultural del sexo (Goldie, 2014). Además, se le
} 
desviaron la atención de discusiones fundamentales sobre la libertad, el retorno a lo «animal» y la posibilidad de «construir(se) (a) un lugar en el mundo» ${ }^{3}$. Es decir, nuestra propuesta apunta hacia la renovación de los debates entre naturaleza y cultura entendiendo que es necesaria la construcción social de lo natural y el cuestionamiento de las propuestas científicas -biológico, médicas- modernas que han pretendido, no necesariamente con éxito, ser estructuras de normativización, normalización y patologización de la «anarquía orgánica» ${ }^{4}$. Es preciso entonces entender el sexo como un producto del género, siguiendo a Judith Butler (1990), y en esta medida podremos construir un sexo diverso y no excluyente que permita la identificación de individuos subjetivados con cuerpos diversos que son los que habitan el mundo contemporáneo.

Desde la segunda mitad del siglo XX los movimientos feministas y la sexología norteamericana han popularizado una falsa dicotomía entre sexo y género, donde se planteaba que el sexo fueran todas las características biológicas relativas a la diferenciación entre hombres y mujeres, y el género los roles sociales asignados a hombres y a mujeres y la identificación individual con dichos roles (Sterling, 2000:18). No obstante, los planteamientos postestructuralistas, el feminismo de la «tercera ola», las teorías queer y los estudios postkuhnianos ${ }^{5}$ han fundamentado el entendimiento de las ciencias y las

ha considerado como «el arquitecto de los protocolos para la asignación quirúrgica de sexo» en infantes intersexuales (Morland, 2014).

${ }^{3}$ Las perspectivas filosóficas a las que se hace alusión al mencionar «lo animal» y el «construir(se) (a) un lugar en el mundo», provienen de las miradas de Jaques Derrida (2006) y Martin Heidegger (1962). Ambos permiten un nuevo entendimiento de las relaciones seres humanos-naturaleza-cultura que invitan a pensar, respectivamente, la (de)construcción de lo animal y la posibilidad de construir diferentes relaciones con el mundo natural desde el situar, el-estar-en.

${ }^{4}$ Sobre el planteamiento de un cuerpo anárquico hemos realizado algunas ponencias e indagaciones. Sin embargo, es un tema que estamos desarrollando actualmente y que será profundizado en posteriores publicaciones. La idea de entender la conjunción entre ciencias e ideologías políticas ha sido ya explorada desde el surgimiento del marxismo. Lo anterior puede evidenciarse por ejemplo en el II Congreso Internacional de Historia de la Ciencia y la Tecnología celebrado en Londres en 1931, al cual por primera vez asistió una delegación de la Unión Soviética que hizo manifiesta la necesidad de preguntarse por «las raíces sociales y económicas» de postulados considerados «universales» como los de Isaac Newton (Needham, 1971). Esta perspectiva de análisis ha sido motivada por los estudios anarquistas realizados en España y en Inglaterra, principalmente por los estudios en torno a la sexualidad (Heckert \& Cleminson, 2011) y al saber médico (Molero, Jiménez, \& Tabernero, 2016).

${ }^{5}$ Como es usual en la historia, la historia del cambio epistemológico para entender la ciencia como construcción social no comienza aquí ni mucho menos, para este artículo hemos mencionado algunos de los ámbitos que han sido más relevantes para nuestras investigaciones. Sin embargo, no podemos dejar de mencionar la importancia del materialismo dialéctico y en general los estudios que se hicieron desde el 
tecnologías como construcciones sociales, lo que ha permitido entender el sexo como una construcción social; un resultado del género, como se mencionó anteriormente, que depende del contexto histórico y geopolítico desde donde se construya. Complementariamente, según los análisis de David A. Rubin, «la intersexualidad jugó un rol principal en la invención del género como categoría en la biomedicina de mediados del siglo XX, y subsecuentemente, en los discursos feministas» (Rubin, 2012:887). Es por lo anterior, que el análisis de la patologización de la intersexualidad las primeras tres décadas del siglo XX es una herramienta fructífera para revisitar los discursos científicos y feministas que se han fundamentado en discursos esencialistas sobre el individuo, e implica revisitar la circulación transnacional de teorías científicas que sobre la sexualidad y los cuerpos sexuados se estaban planteando en la época.

Alrededor de la idea de normativizar el cuerpo no podemos olvidar la historiografía que se ha consolidado sobre la eugenesia, el degeneracionismo y la institución de los EstadosNación, tanto europeos como latinoamericanos (aunque en diferentes momentos históricos). Dichos ámbitos inmediatamente evocan los conceptos foucaultianos de biopoder y biopolítica (Foucault, 1977, 1984, 1987). En Colombia durante la segunda década del siglo $\mathrm{XX}$, hubo un corto pero intenso debate sobre los temas mencionados, enfrentando posiciones sobre las dimensiones sociales de la salud y la enfermedad contra las ideas biologicistas y hereditarias de la constitución orgánica ${ }^{6}$. Este contexto proteccionista y que encarnaba las ideas de progreso y de modernidad aportaría su parte para que los discursos médicos generaran nuevos discursos sobre la sexualidad, la maternidad y el desarrollo de los individuos, así como nuevas representaciones de las mujeres, de la infancia (Pedraza,

marxismo sobre las ciencias y las tecnologías desde la tercera década del siglo XX, así como la importancia del giro lingüístico de la segunda mitad del mismo siglo.

${ }^{6}$ Los debates historiográficos actuales sobre la eugenesia en Colombia tienen muchos matices que no se plantearán en este artículo. Uno de los principales radica en la pregunta de si se debe hablar de eugenesia en Colombia a principios del siglo XX o más bien de degeneracionismo, las investigaciones mencionadas a continuación versan sobre ambas vertientes. Para profundizar en los debates sobre eugenesia, la consolidación del Estado Colombiano y el concepto de raza véase el trabajo de Álvaro Andrés Villegas (2005); sobre la relación con la implementación de programas pedagógicos en escuelas y colegios y la relación con el «Proyecto Moderno» véase Andrés Klaus Runge y Diego Alejandro Muñoz (2005) y Carlos Arturo Ospina y Andrés Klaus Runge (2016); la reciente tesis doctoral de Juan Vianey Tovar (2016) realiza un análisis sobre proyectos legislativos formulados en dicha época; y finalmente, acerca del el reforzamiento de un movimiento higienista organizado puede consultarse el artículo de Jason McGraw (2007). 
1997), y de las personas con sexualidades no heteronormativas. Las teorías de Spencer, Morel y Lombroso; el darwinismo y el neolamarkismo, fueron ideas que circularon por el territorio colombiano por esta época y fueron determinantes para los discursos y prácticas que los médicos consolidaron para la normativización de los cuerpos intersexuales.

Igualmente, para entender los discursos médicos que sobre la intersexualidad se consolidaron en esta época es preciso preguntarse por los cambios sociales en torno al concepto de infancia. No por coincidencia, fue en este momento en el que se legitimó la institucionalización de la pediatría y la obstetricia colombiana ${ }^{7}$. Algunas investigaciones sobre los transformaciones jurídico, médicas y educativas que se dieron a principios del siglo XX colombiano concernientes a los niños y a las niñas plantean por ejemplo, que «el niño de las colonias fue subordinado y asumido como recurso» e incorporados en el concepto de lo moderno (Amador, 2009), que comenzaron a entenderse como «individuos económicamente rentables» (Garcés, 2017:309), así como agentes de consumo (Aristizábal, 2016). Los médicos comenzaron a construir la normalidad de los infantes a través no sólo de la determinación de la «anormalidad» o lo «patológico», sino también de la construcción de herramientas cualitativas que implicaban propuestas normativas para clasificar los cuerpos $^{8}$. Lo anterior, influenciado por diversas corrientes científicas, como la eugenesia y la biotipología, circularon por el territorio latinoamericano nutriendo y nutriéndose de las perspectivas europeas de finales del siglo XIX y principios del XX. Desde principios del siglo XX, en Colombia se comenzó a medicalizar la niñez, en diferentes ciudades colombianas, como Medellín, Cali, Barranquilla, Manizales, Cartagena y Bogotá dónde se fundaron hospitales infantiles que marcaron el comienzo de la profesionalización pediátrica en el país (Bonilla, 2013). En 1940, se evidenció la necesidad de nacionalizar el proceso de institucionalización de la pediatría y la obstetricia colombiana, hasta hora realizada a nivel regional. El establecimiento de la Sociedad Colombiana de Pediatría y de la Sociedad Colombiana de Obstetricia y Ginecología dan cuenta de ello (Editorial, 1943) (Sánchez, 1943).

\footnotetext{
${ }^{7}$ En octubre de 1942 la Sociedad Colombiana de Pediatría aprobó sus estatutos (Editorial: Nuestras relaciones pediátricas, 1943) y en abril de 1943 se realizó el acto inaugural de la Sociedad Colombiana de Obstetricia (Sociedad de Obstetricia, 1943).

${ }^{8}$ Para profundizar en las nociones sobre «lo normal y lo patológico», véase: (Canguilhem, 1971).
} 
Cuando hablamos de historia de la intersexualidad, uno de los problemas metodológicos que se presentan es el rastreo de información. El mismo proceso de medicalización de la infancia y de patologización de la intersexualidad en Colombia se evidencia en la poca presencia de referencias a personas intersexuales durante el principio del siglo XX. Sin embargo, en las revistas de especialidades médicas como la pediatría y la obstetricia se puede dar cuenta de un creciente interés por temas como la sexualidad infantil, la «predeterminación del sexo» y el «diagnóstico del sexo» durante el embarazo, la puericultura, la circuncisión, la amenorrea, el síndrome de Laurence-Bardet-Biedl, la homosexualidad, «patologías» de los genitales femeninos y masculinos, la precocidad sexual, temas que nos permiten develar categorías determinantes de los parámetros de normalidad utilizados por los médicos colombianos para demarcar el ser mujer y el ser hombre desde el nacimiento. La información de esta investigación se ha rastreado principalmente en la Revista Colombiana de Pediatría y Puericultura entre los años 1942 y 1961, la Revista de la Sociedad Colombiana de Pediatría y Puericultura entre 1959 y 1962, Anotaciones Pediátricas entre 1954 y 1960, Medicina y Cirugía de Bogotá entre 1939 y 1956 y la Revista Colombiana de Obstetricia y Ginecología (Bogotá) entre 1950 y 1953 y el año 1959. Además, se encontraron algunos artículos relacionados en el Índice de la literatura médica colombiana 1890-1960 realizado en 1965 (Céspedes de Bayona et al., 1965), que abarcan un periodo un poco más amplio del que se exploró en las revistas mencionadas. Este proceso nos permitió identificar a algunos médicos interesados en definir «lo normal» y «lo patológico», sobre todo en la infancia, y analizar algunas perspectivas planteadas por ellos para comenzar a develar parte del discurso patologizante que se construía sobre la intersexualidad en la época.

El tránsito de los conocimientos científicos (Secord, 2004) es fundamental para entender la constitución de discursos locales que se han consolidado en tensión con las «luchas por los significados» (Jiménez et al., 2014). Luchas que incluyen relaciones de poder entre marañas interseccionales de múltiples interfaces sociales y culturales ${ }^{9}$. Es por la anterior

\footnotetext{
${ }^{9}$ Es importante plantear que la conjunción entre el discurso interseccional y de las interfaces tecnológicas es una reciente aproximación en la cual nos encontramos trabajando, por lo que no queremos hacer referencia determinante a un referente teórico específico.
} 
que para entender la historia de la patologización de la intersexualidad en Colombia, debemos remitirnos al análisis de discursos científicos que fueron hegemónicos para el discurso médico colombiano y a las maneras en que este último se apropió y transformó dichos discursos. El presente artículo se centrará en la circulación en Colombia de las teorías del endocrinólogo español Gregorio Marañón y Posadillo (1887-1960) sobre «los estados intersexuales», principalmente a través de las discusiones planteadas por el médico endocrinólogo Francisco Gnecco Mozo (1904-1973) en Bogotá a su regreso de Madrid después de haber estudiado con Marañón en el Instituto de Patología del Hospital Provincial de Madrid (Jácome Roca, 2004) durante el curso $1932-1933^{10}$. Las ideas de Francisco Gnecco Mozo planteaban interrogantes a las representaciones que los médicos colombianos estaban realizando sobre los cuerpos sexuados e intersexuales en la primera mitad del siglo XX, así como a los planteamientos marañonianos. Igualmente, se plantearán dos contribuciones epistemológicas para entender la patologización de la intersexualidad evidenciadas en el discurso médico colombiano y sobre las cuales esperamos seguir profundizando durante la realización de este proyecto de investigación.

\section{Gregorio Marañón y Posadillo y los parámetros de normalidad en el discurso médico colombiano. Diferenciación sexual e intersexualidad}

Gregorio Marañón y Posadillo ha sido un renombrado personaje de la historia de España y de la historia de la medicina particularmente. Ya desde 1976 el historiador de la medicina Thomas F. Glick lo describió como el único impulsor de la endocrinología en España, así como un liberal conservador que participó activamente de las luchas sociales de la época promoviendo la igualdad de hombres y mujeres (Glick, 1976). En el camino historiográfico que se ha hecho sobre la figura de Gregorio Marañón existen varios tipos de análisis:

\footnotetext{
${ }^{10}$ El paso de Francisco Gnecco Mozo por el Instituto de Patología de Madrid en el que Gregorio Marañón impartía sus clases, puede evidenciarse en el artículo de Gnecco Mozo La sexualidad general y las glándulas genitales publicado en 1940, en el que cita de manera directa sus notas de clase tomadas en 1932. Asimismo, en el año 1933 Gregorio Marañón escribió el prólogo del libro de Francisco Gnecco titulado El trabajo del Corazón en Bogotá publicado en la misma ciudad por la Editorial Cromos, y en ella hace alusión a la presencia del médico colombiano en dicho instituto durante el año mencionado.
} 
aquellos que buscan homenajearlo por su trayectoria médica, política y en general por su prolífica producción escrita; aquellos que lo visualizan como un liberal impulsor del movimiento feminista de los años 20 y 30 y buscan mostrarlo como un «transgresor», «pionero», «revolucionario» de los roles asociados a hombres y mujeres; y por último, los que buscan entender históricamente su producción científica, ya sea de manera descriptiva sobre sus publicaciones o posicionándose críticamente ante ellos ${ }^{11}$. Para el tema que nos compete en este artículo, es preciso plantear que Gregorio Marañón publicó una extensa teoría sobre el sexo, el género y la vida sexual de hombres y mujeres. Sus ideas contenidas en la obra La evolución de la Sexualidad y los Estados Intersexuales ${ }^{12}$, publicada por primera vez en 1929, fueron exiguamente modificadas durante su carrera profesional y constituyeron la base para las propuestas científicas, principalmente endocrinológicas, que marcaron su acción clínica e investigadora.

Desde 1920, Gregorio Marañón dejó clara su posición frente al feminismo, aunque promovía la consideración de las mujeres como ciudadanas y sujetos de derecho, fundamentaba de manera conservadora, católica y sobre todo determinista $\mathrm{y}$ heteronormativa la diferenciación sexual y la asignación de roles sociales según características biológicas específicas de hombres y mujeres, clarificando siempre que las mujeres que querían plantearse vidas académicas o profesionales presentaban una «masculinización anormal» (Marañón y Posadillo, 1920).

Las teorías marañonianas sobre «los estados intersexuales» no versan únicamente sobre la intersexualidad como categoría nosológica. Marañón, a través del uso de la idea de la potencialidad del tejido embrionario para desarrollarse en cualquiera de los sexos y recogiendo la teoría de la recapitulación, planteó un ideal de género que describe onto-

\footnotetext{
${ }^{11}$ Se puede profundizar en el análisis histórico referente a la obra de Gregorio Marañón dirigiéndose al dossier publicado recientemente en la revista Arbor, Vol 189, No 759 (2013), bajo el título: Gregorio Marañón y Posadillo (1887-1960). Cincuenta años después (Balaguer Perigüell y López Vega, 2013). Una obra de consulta imprescindible sobre la figura de este médico español es la ya bastante conocida recopilación y análisis que realizó Pedro Laín Entralgo a finales de los años ‘60s (1967), (1969).

${ }^{12}$ Es preciso aclarar que para Gregorio Marañón el término intersexual no significaba lo mismo que significa en la actualidad, para él «estados intersexuales» son aquellos estados «intermedios» entre hombres y mujeres. Al plantear la diferenciación total entre hombres y mujeres como un ideal, precisamente su «ideal evolutivo», se puede llegar a la conclusión de que prácticamente todas las personas seríamos intersexuales. Cuando Gregorio Marañón se refería a las personas con diversidad genital utilizaba el término hermafroditismo (Marañón y Posadillo, 1930).
} 
filogenéticamente como un desarrollo-evolución que parte de un ser de sexualidad indiferenciada infantil-primitivo hacia una diferenciación total entre hombres y mujeres, que sería el estado de madurez-evolución;

La base de nuestro razonamiento y que considero esencial dejar sentada desde el primer momento, a saber: que, como ya hemos dicho, entre los signos físicos de la feminidad y los de la masculinidad no existe una oposición radical, sino tan sólo la diferencia cuantitativa correspondiente a dos fases sucesivas de un único proceso evolutivo (Marañón y Posadillo, 1930:40, cursivas del autor).

Así y todo, en cuanto Marañón intenta presentarnos idealmente una equiparación entre hombres y mujeres y que ambos tenemos características biológicas de ambos, cuando describe los rasgos de indiferenciación asemeja estos a los de la mujer: «cuando falta la secreción genital -estado eunucoide- la pelvis queda en un estado indiferenciado, más próximo al de la mujer que al del hombre» (Marañón y Posadillo, 1930:17). Planteando que en algunos caracteres el estado de perfección es el masculino y que la mujer es por definición, para esos mismos caracteres, un estado imperfecto, indiferenciado, infantil: «[...] una zona intermedia entre la adolescencia y la masculinidad» (Marañón y Posadillo, 1930:158). No sorpresivamente los caracteres sexuales que para Marañón son los evolucionados en la mujer son los referentes a la maternidad:

...por fin las mamas, rudimentarias en el hombre y desenvueltas en la mujer, hacen también excepción a la ley general del mayor desarrollo varonil. [...] dentro de su general hipoevolución, el organismo femenino se ha especializado para una función determinada, que es la maternidad (Marañón y Posadillo, 1930:42).

Esencialmente, Gregorio Marañón planteó una teoría científica que le permitió determinar biológicamente cuales son los roles sociales para los cuales mujeres y hombres serían $\operatorname{aptos}^{13}$.

La intersexualidad, entendida como diversidad genital, y la homosexualidad eran para Gregorio Marañón factibles dentro su teoría sobre la sexualidad. Podemos hablar de ellas conjuntamente porque dentro del determinismo de Marañón ambas serían diferentes grados de lo mismo, en términos orgánicos, sin negar la influencia de «causas externas». Sin

\footnotetext{
${ }^{13}$ La construcción de la epistemología determinista y teleológica de Gregorio Marañón amerita un análisis detallado; las metáforas y argumentaciones utilizadas por él para conciliar el desarrollo embrionario, la evolución y sus posturas ideológicas, su conservadurismo liberal y su catolicismo, son amplias y requieren un análisis específico, es por esto que serán detalladas en una publicación posterior.
} 
embargo, Gregorio Marañón estableció parámetros normativos, al estilo de la biotipología de Nicola Pende, para categorizar los cuerpos entre normales y anormales, y construyó los límites entre lo normal y lo patológico refiriéndose a la intersexualidad y a la homosexualidad como «accidentes naturales», «perturbación sexual», «instinto torcido», «manifestaciones aberrantes», «casos de intersexualidad graves», entre otras etiquetas. Otra evidencia del determinismo biológico de Marañón era su concepción de que «la diferenciación sexual» podía «favorecerse» por medios terapéuticos, como la «opoterapia y los injertos», y que por tanto la homosexualidad podía ser curada, en cuanto a la intersexualidad -a la que él se refería como hermafroditismo- la consideraba irremediable (Marañón y Posadillo, 1930:250-266).

Francisco Gnecco Mozo, fue nombrado profesor de la cátedra de endocrinología de la Facultad de Medicina de Bogotá en $1937^{14}$. En 1940, en su artículo La sexualidad genital y las glándulas genitales describió los debates que sobre la sexualidad y la diferenciación entre hombres y mujeres se hacía la comunidad médica colombiana. En un momento en el que desde las «glándulas virilizantes» y las «glándulas feminizantes» se estaba construyendo una diferenciación fija y categórica entre hombres y mujeres, Francisco Gnecco manifestó su prevención ante los discursos categóricos sobre el dimorfismo sexual y el «determinismo genital» (Holmes, 2008):

Nosotros no aceptamos la clasificación así propuesta, no solo porque de los hechos patológicos no hay que sacar conclusiones fisiológicas permanentes, sino porque aun cuando fuera lógico hacerlo, la llamada virilización de la mujer, por ejemplo, en la acromegalia consiste esencialmente en la amenorrea, tan frecuente en todos los desarreglos hormonales, en la voz grave, que podría atribuirse al mayor tamaño de la laringe de las acromegálicas, y en los rasgos fisonómicos como el prognatismo maxilar, alargamiento de la nariz, etc., que si pueden llamarse masculinos es apenas por su fealdad (Gnecco Mozo, 1940:140-141).

Para problematizar el genital-centrismo, Gnecco Mozo comenzó por plantear el papel del sistema nervioso y las glándulas endocrinas, principalmente la hipófisis, en la sexualidad.

\footnotetext{
${ }^{14}$ Gnecco Mozo, F. (1937). Correspondencia Gregorio Marañón (CA4, S20, 1), Archivo de la Fundación José Ortega y Gasset-Gregorio Marañón.
} 
La castración y la evidencia de personas con una sexualidad «normal» después de castradas supone para Gnneco Mozo la principal prueba de que existe algo más determinante de nuestro desarrollo sexual que los genitales. Del mismo modo, desde el punto de vista hormonal, planteó una problematización al discurso categórico y dicotómico de la diferenciación entre hombres y mujeres según «la acción bisexual de las hormonas» (Gnecco Mozo, 1940:149), legitimando el discurso de Gregorio Marañón y las relaciones de éste con las concepciones galénicas:

Si bien es cierto que las nuevas adquisiciones han traído ciertos visos de comprobación de teorías modernas sexuales, como la de la «Evolución de la Sexualidad» de Marañón, ya que la mayor complejidad de la hormona masculina está de acuerdo con la escala que va desde el infantilismo hasta la masculinidad, pasando por el escalón aún imperfecto de la feminidad, no es menos de verdad que con las adquisiciones nuevas, el «problema hormonal» resulta aún más complicado que antes. (...) La solución de los problemas que hemos expuesto, están muy cerca aún de la concepción de Galeno: «todas las partes que se encuentran en el hombre pueden verse también en el cuerpo de la mujer» tan de acuerdo con las más aceptadas nociones modernas respecto a los caracteres secundarios, que en vez de hacerlos contrarios para los dos sexos los considera apenas como etapas de desarrollo en la evolución sexual (Gnecco Mozo, 1940:149-150).

El médico Gnneco Mozo realizó en el mismo artículo una distinción sobre la «naturaleza» de hombres y mujeres para comenzar a disertar sobre el «hermafroditismo». Después de hacer alusión a la diferenciación de los caracteres físicos realizó algunas metáforas dualistas a las que se refirió como:

caracteres sexuales secundarios de orden espiritual (...) se ha señalado en la mujer una tendencia a la pasividad, mayor emotividad, altruismo elevado, el amor maternal, etc., etc., en el hombre hay, en cambio, menos sensibilidad afectiva, mayor capacidad de abstracción mental y de creación, etc» (Gnecco Mozo, 1940:142).

Caracteres que de manera determinista, igual que Gregorio Marañón, atribuyó a «la actividad de las glándulas genitales» y a la carencia o exceso de un tipo hormonal: «pero esto de que en una mujer aparentemente ciento por ciento, se encuentren glándulas genitales masculinas, no tendría otra explicación de la que un exceso de hormonas de un 
sexo fuera capaz de provocar caracteres secundarios del sexo opuesto» (Gnecco Mozo, 1940:143). Tras realizar dicha diferenciación entre hombres y mujeres, calificó de «increíbles y excepcionales», «casos raros» o «curiosidades científicas» los hallazgos de personas intersexuales de Robert Meyer y de Gregorio Marañón, aun cuando aludió a ciertos casos que él mismo había tratado como pacientes.

Vale la pena resaltar que el artículo de Francisco Gnecco trataba de plantear una explicación de la relación entre sexualidad y las glándulas genitales para superar el discurso centrado en los genitales, desde «la normalidad» fisiológica. Así como Gregorio Marañón, cuando se refería a la intersexualidad no planteaba estos estados como patologías, sino como estados graduales del sexo; «el hermafroditismo nos ofrece toda una gama de combinaciones de los caracteres sexuales secundarios, la mayor parte de los cuales se explican por la supremacía de la acción de una glándula genital entre las dos coexistentes» (Gnecco Mozo, 1940:143-144). Finalmente, concluyó que es necesario definir la importancia de la «preponderancia de unas hormonas sobre las otras» y de la herencia genética para poder explicar la diferenciación sexual, el ser «apenas más o menos hombre o más o menos mujer», proponiendo un movimiento hacia un determinismo genético:

(...) tenemos que contentarnos con que su influencia [el de las glándulas genitales] en la diferenciación sexual se limita a proteger el sexo, a desarrollar los caracteres sexuales ya preexistentes, desde la fecundación del individuo. Desde el momento en que el espermatozoide fecunda el óvulo, ya está decidido por el determinismo de la herencia si el futuro ser humano tendrá ovarios o testículos, o al menos, cuál de estas dos glándulas genitales será la preponderante; allí mismo surge el esbozo de diferenciación sexual que impregna todas las células del cuerpo, y en aquella célula fecundada primitiva se halla también la estructura cromosomal origen del pene o de la vulva, del rostro lampiño o de la barba hirsuta, de la voz de soprano o de la grave, del amor maternal o del impulso guerrero!... (Gnecco Mozo, 1940:145).

Contrario a los postulados de Gregorio Marañón, Francisco Gnecco no definió la homosexualidad desde el ámbito médico, biológico sino desde el psicológico. Propuso la necesidad de una demarcación entre homosexuales, «pervertidos sexuales», e intersexuales, haciendo manifiesto el diagnóstico para él errado de «hermafroditismo psíquico». La 
confusión entre intersexualidad y homosexualidad en la época la atribuyó a la consideración de los homosexuales como intersexuales, planteamiento que es claro y fundamental en las teorías marañonianas. Esta demarcación pareciera deberse más a una necesidad de realizar una demarcación disciplinar entre psiquiatría y endocrinología:

lo característico de la homosexualidad es la inversión del impulso sexual, fenómeno psíquico, y el hecho de su estudio se salga más de los límites de la psiquiatría para abarcar el campo endocrinológico se debe precisamente a confusión con el hermafroditismo, que hace invertir la influencia de las hormonas sexuales (Gnecco Mozo, 1940:147).

Por lo tanto, concluye que el tratamiento hormonal no servirá para «curar» la homosexualidad, pero sí la intersexualidad.

\section{Contribuciones epistemológicas en el discurso médico colombiano para patologizar la intersexualidad}

Siguiendo el análisis de la circulación de las teorías de Gregorio Marañón en el territorio colombiano, y por lo tanto en los discursos médicos que allí se construyeron, a continuación delinearemos dos contribuciones epistemológicas que hemos encontrado fundamentales para el proceso histórico de la patologización de la intersexualidad, lo que además está estrechamente relacionado con la reafirmación de la heteronormatividad. Estas contribuciones epistemológicas son: la insistencia en que lo femenino permanezca femenino y la invención de síntomas para personas intersexuales, síntomas que no eran tales si aparecían en personas «normales».

El programa feminista de Marañón se basaba en la diferenciación como forma de acabar con la inferioridad de la mujer, en sus palabras:

Hacer muy hombres a los hombres y muy mujeres a las mujeres. En esto estribará la liberación de éstas, y sobre este eje ha de construirse el programa del feminismo verdadero... y es evidente que, tanto para el progreso individual como por el auge colectivo del sexo, hay que empezar por substituir este sentimiento de inferioridad por el cultivo, la diferenciación y el ennoblecimiento de la feminidad por sí misma, purificada de todo virilismo, no convergiendo hacia éste, sino paralela a él (Citado por: Castejón, 2013:6). 
Ya desde 1924 en Colombia, se veía una intención de definir lo femenino como una característica necesaria para determinar la normalidad. El médico Gil J. Gil, caracterizaba a sus pacientes con «anomalías congénitas del aparato genital femenino» como «tipo femenino franco» cuando algunos genitales se presentaban de manera «dudosa», «rudimentarios», con «falta de desarrollo» o con ausencia de vagina y/o útero (Gil, 1924:118); y «tipo femenino neto» cuando las mujeres tenían los genitales femeninos para él «normales»(Gil, 1924:117). En cuanto a la intersexualidad puede evidenciarse que algunos médicos como Miguel Barreto promovían el «urgente» tratamiento hormonal para cuidar de que la feminidad permaneciera como tal, mas no para evitar una sobremasculinización de una persona intersexual definida por él como hombre:

(...) el tratamiento de la hiperplasia es de mayor urgencia en la mujer que en el varón; éste toma la apariencia de un adulto normal cuando le llega la edad puberal, aun cuando su fertilidad es aún dudosa: en la mujer hay tendencia a permanecer con sus caracteres masculinoides. Si estos pacientes no se tratan, aun cuando su crecimiento es rápido en sus primeras etapas, cesa luego completamente a muy temprana edad debido a la soldadura de las epífisis, haciéndoles quedar de pequeña estatura. A los niños a quienes se refiere esta comunicación se les ha seguido observando en la consulta externa, en donde la niña seguirá tratamiento hormonal (Barreto, 1945:223).

Más allá de las aportaciones de los médicos colombianos al «proyecto del dimorfismo sexual», éstos construyeron signos clínicos para definir la intersexualidad que en discursos médicos de la misma época habían sido características intrínsecas de «la raza» colombiana. Así la «hipersexualidad» y la «agresividad» eran consideradas signos diagnósticos de la intersexualidad. Por ejemplo, en 1945 el médico Miguel Barreto, publicó en la Revista Colombiana de Pediatría y Puericultura un artículo titulado: «Síndrome adrenogenital y fisiología de la glándula suprarrenal. Comunicación del caso de un par de hermanos», en el cual realizó una descripción de los síntomas del síndrome adrenogenital. Uno de los conjuntos de síntomas descritos en dicho artículo fue denominado «trastornos psico- 
fisiológicos», y lo describió como «generalmente más agresivos y esforzados que sus contemporáneos, y presentan frecuentes erecciones, pudiendo originar pequeños problemas sociales. Ya veremos por ejemplo, como nuestro paciente, de solo dos años de edad, se complace en mostrar sus órganos genitales». Sin embargo, más adelante en su artículo defiende que los «trastornos psico-fisiológicos» se podían comparar con la pubertad «normal»: «Al llegar a la edad puberal, los síntomas pueden confundirse en cierto grado con los de una pubertad normal más o menos precoz» (Barreto, 1945:206). Refiriéndose específicamente a los casos mencionados, el médico Miguel Barreto planteó la «enfermedad» del niño de dos años desde la «normalidad»:

(...) la madre dice que el vello (púbico) no le ha aumentado mayormente, pero que el crecimiento del pene ha sido gradual y progresivo, presentando frecuentes erecciones, sin eyaculación, durante las cuales alcanza el tamaño del dedo índice de un adulto; y agrega, que no le gusta entretenerse con juguetes si no que parece más bien divertirse mostrando los órganos genitales. Por lo demás sus juegos y actividades son normales. (...) A la inspección se encuentra un niño de aspecto sano, robusto, de musculatura fuerte, de buen semblante, vivaz. Que juega con su madre pero se transforma en un peleadorcito fuerte, llorón y gritón cuando se trata de examinarlo (Barreto, 1945:212, 214).

Surge entonces la pregunta: ¿pueden la «agresividad» y la «hipersexualidad» considerarse rasgos de la personalidad que devinieron síntomas para aportar a la construcción de la intersexualidad como enfermedad? El concepto de «hipersexualidad», se utilizó por el médico Álvaro Navia Monedero como una característica de la sociedad colombiana; la definió en la infancia como unas «tempestades de sexo» (Navia Monedero, 1957:426) y una «obsesión pertinaz y tozuda» por indagar y explorar el sexo (Navia Monedero, 1957:429). Dicho médico entendía la «hipersexualidad» desde dos dimensiones: la «hipersexualidad psicológica» y la «hipersexualidad constitucional» u «orgánica». La hipersexualidad constitucional, orgánica, deriva de otros factores no dependientes de la educación (...) Entre estos factores el de la raza ha sido siempre punto de apoyo para justificar nuestra conducta sexual, ya que nuestras razas de origen india, española y negra, son de hipersexuales, [es decir la sociedad colombiana tendría una] constitución ancestral de hipersexuales (Navia Monedero, 1957:427). 
Según el discurso médico de la época, la hipersexualidad psicológica se construía por el ocultamiento de la sexualidad y por las «informaciones defectuosas» que se daban a infantes sobre este tema, es decir, se consideraba producto de la concepción del sexo como tabú. Dado esto, la «naturalización» del sexo y de la sexualidad, la explicación de ellas desde la «verdad médica», serían la «cura» de esta característica indeseable; «(...) así procederíamos de forma análoga a como lo hicieron con nosotros cuando niños "nos

prohibían escuchar las conversaciones de los mayores" y nos hipersexualizaban» (Navia Monedero, 1957:439). Finalmente, puede verse entonces como, aunque el mismo médico se considerase «hipersexual», esto no suponía un signo patológico, sino más bien una característica intrínseca de la «raza colombiana», que podría ser «corregida» a través de la desmitificación de la sexualidad por «la verdad científica» que se estaba consolidando en los discursos médicos colombianos de la primera mitad del siglo XX.

\section{Conclusión. Patologización de la intersexualidad en Colombia: de la disforia de género a la discapacidad sexual}

Los debates sobre la intersexualidad en Colombia se retomaron por un hito histórico fundamental que puso en auge la discusión sobre intersexualidad a nivel nacional e internacional; en 1999 la Corte Constitucional Colombiana falló a favor del consentimiento informado de infantes mayores de cinco años, restringiendo la autoridad legal de la madre y el padre para autorizar cirugías de reconstrucción genital. Sin embargo, acudiendo al principio de dignidad y a su definición de «contexto cultural colombiano», la corte imputó como única posibilidad para las personas intersexuales identificarse como hombres o mujeres olvidándose de los principios de pluralismo y de igualdad con que nacen lxs ciudadanxs colombianxs (Velásquez et al., 2007).

En el 2012, por iniciativa de la Dirección de Diversidad Sexual de la Secretaria Distrital de Planeación de Bogotá y ejecutado por el Centro de Estudios de Género de la Universidad 
Nacional de Colombia se realizó el Diagnóstico de Personas Intersexuales en la ciudad, con el fin de evidenciar las necesidades de las personas intersexuales que deben ser reclamadas ante la administración pública. El cambio de la denominación política de la diversidad sexual de LGBT a LGBTI ha suscitado la revisión académica, política y social de la necesidad de demarcación cultural de las personas intersexuales para posibilitar la claridad y especificidad de los cambios que deben realizarse en las políticas públicas de Colombia para atender sus necesidades y definir las diferencias que existen entre, principalmente transexuales e intersexuales. Vale la pena resaltar, que las propuestas generadas a partir de esta investigación para enfrentar las problemáticas identificadas lograron ser incluidas en el Plan de Desarrollo Distrital 2012-2016. Dicho diagnóstico parte de premisas como la construcción social del sexo, la «mitología de lo normal» de las disciplinas médicas que perpetúan el modelo dicotómico hombre-mujer y la presencia innegable de «cuerpos disidentes» que transgreden dichas categorías de normalidad. Sin embargo, llama la atención que las propuestas realizadas a la administración pública no sean hacia el repensar categorías incluyentes de las políticas públicas de la salud, sino a tratar de enmarcar otra vez a las personas intersexuales en una categoría fija y bastante problemática, la «discapacidad sexual» (Prada, 2012). Porque como ya ha manifestado públicamente Hilda Viloria fundadora de la «Organization Intersex International», la discapacidad es creada por la mutilación genital y las intervenciones médicas y no por ser intersexuales (OII Intersex network, 2003).

Es necesario conocer los procesos mediante los cuales se construyó la intersexualidad como patología para poder deconstruir esta categorización estructurante y peligrosa. En la medida en que se devele que dichos procesos se construyen desde planteamientos subjetivos; culturales, políticos e históricos, podremos potenciar tecnologías individuales que permitan «revoluciones moleculares» (Guattari y Rolnik, 2005) de cuerpos artefactos construidos consciente e individualmente para, como diría Beatriz Preciado, construir esa libertad que no existe (Preciado, 2003). 


\section{Bibliografía}

Amador, J:C. (2009) La subordinación de la infancia como parámetro biopolítico y diferencia colonial en Colombia (1920-1968). Nómadas (31), 241-256.

Aristizábal, D. M. (2016). Niños deseantes y mercados emergentes. Reflexión histórica sobre la infancia y el consumo en Colombia, primera mitad del siglo XX. Trashumanante. Revista Americana de Historia Social (8), 200-225.

Balaguer Perigüell, E. y López Vega, A. (2013). Gregorio Marañón y Posadillo (18871960). Cincuenta años después. Arbor 189(759), a001.

Barreto, M. (1945). Síndrome adrenogenital y fisiología de la glándula suprarrenal, Comunicación del caso de dos hermanos. Revista Colombiana de Pediatría y Puericultura $6(5), 198-225$.

Bonilla, E. (2013). La cirugía pediátrica, una obra de caridad. Revista Colombiana de Cirugía (28), 266-270.

Bullough, V. L. (2003). The contributions of John Money: A personal view. The Journal of Sex Research 40 (3), 230-236.

Butler, J. (1990). Gender trouble: feminist and the subversion of identity. New York: Routledge.

Canguilhem, G. (1971). Lo normal y lo patológico. Buenos Aires: Siglo XXI.

Castejón Bolea, Ramón (2013) Marañón y la identidad sexual: biología, sexualidad y género en la España de la década de 1920. Arbor 189(759), a005:1-9.

Céspedes de Bayona, B. et al. (1965). Índice de la literatura medica colombiana 18901960. Bogotá: Asociacion Colombiana de Facultades de Medicina. 
Derrida, J. (2006) El animal que luego estoy si(gui)endo. [Traducción al español: de Parelti, C. y Rodriguez Marciel, C.] (2008) Madrid: Editorial Trotta, S. A.

Editorial. (1943). Revista Colombiana de Pediatría y Puericultura 2(5), 194-195.

Foucault, M. (1976). Historia de la Sexualidad I. La Voluntad del Saber. [Traducción al español: Guiñazú, Ulises] (2005) Buenos Aires: Siglo XXI.

Foucault, M. (1984). Historia de la Sexualidad II. El uso de los placeres. [Traducción al español: Marí, Soler] (2012) Madrid: Editorial Biblioteca Nueva, S. L.

Foucault, M. (1984). Historia de la sexualidad III. La inquietud de sí. [Traducción al español: Segovia, Tomus] (1987) México: Siglo XXI.

Garcés, J. D. (2017). "El delicuente de hoy, será el obrero de mañana". Políticas de la infancia y trabajo: instituciones, discursos, prácticas en Colombia (1920-1940). Historia y Sociedad (32), 285-315.

Gil, G. J. (1924). Anomalías congénitas del aparato genital femenino. Revista de la Clínica Gil 1(5), 117-120.

Glick, T. F. (1976). On the diffusion of a new speciality: Marañón and the "crisis" of Endocrinology in Spain. Journal of History of Biology 9(2), 287-300.

Gnecco Mozo, F. (1940). La sexualidad general y las glándulas sexuales. Revista de la Facultad de Medicina (Bogotá) 9(3), 138-150.

Goldie, T. (2014). The man who invented gender: engaging the ideas of John Money. Vancouver: University of British Columbia Press.

Guattari, F. y Rolnik, S. (2005) Micropolítica. Cartografías del deseo. [Traducción al español de: Gómez, F.] (2006) Madrid: traficantes de Sueño.

Heidegger, M. (1962) Ser y tiempo. [Traducción al español de: Gaos, J.] (1983) México: F:C:E.

Heckert, J., \& Cleminson, R. (2011). Anarchism \& Sexuality. Ethics, Relationships and Power. Londres y Nueva York: Routledge. 
Holmes, M. (2008). Intersex: a perilous difference. Selinsgrove: Susquehanna University Press.

Jácome Roca, A. (2004). Revista de Medicina. Bogotá: Academia Nacional de Medicina, Ministerio de Educación Nacional, [En línea]. Consultado el 20 de febrero de 2015 en: revistamedicina.net/weboficial/images/documentos/DiabetesenColombia1.pdf

Jiménez Lucena, I. et al. (2014). The struggle for meaning in Q\&A sections of the Spanish press in the first third of the 20th Century. The anarchist-libertarian and bourgeoisconservative experiences. International Workshop Science as Cultural Hegemony: Gramscian Concepts for the History of Science. Barcelona.

Kessler, S. J. (1990). The medical construction of gender: case managment of intersex infants. Signs 16 (1), 3-26.

Laín Entralgo, P. (1967). Gregorio Marañón. Obras completas. Madrid: Espasa-Calpe.

Laín Entralgo, P. (1969). Gregorio Marañón. Vida, obra y persona. Madrid: Espasa-Calpe. Marañón y Posadillo, G. (1930). La Evolución de la Sexualidad y Los Estados Intersexuales. Madrid: Morata.

Marañón y Posadillo, G. (1920). Biología y Feminismo, conferencia pronunciada en la Sociedad Económica de Amigos del País, de Sevilla, el 21 de febrero. En Juderías, A. (Comp.) (1972) Gregorio Marañón. Obras Completas, Madrid: Espasa-Calpe, S.A., Tomo III Conferencias: 9-33.

McGraw, J. (2007). Purificar la nación: eugenesia, higiene y renovación moral-racial de la periferia del Caribe Colombiano, 1900-1930. Revista de Estudios Sociales (27), 62-75.

Molero, J. et al. (2016). Subverting body regulations: Eugenics and neo-Malthusianism in early 20th century Spanish anarchist press. En A M. Proietto y T. C. Ribeiro (Comp.), Book of Abstracts. Joint Meeting ESHHS \& CHEIRON (p. 181-182). Barcelona.

Morland, I. (2014). Intersex. Transgender Studies Quartterly 1(1-2), 111-115.

Navia Monedero, A. (1957). Introducción a la educación sexual en la infancia. Revista de la Sociedad Colombiana de Pediatría y Puericultura 16(4), 426-443. 
Needham, J. (1971). Foreword. A Science at the Cross Roads; Papers Presented to the [2nd] International Congress of the History of Science and Technology Held in London from June 20th to July 3rd, 1931 by the delegates of the U.S.S.R. London: Russian Foreing Laguages Press Kniga: 1-9.

OII Intersex network. (2003) [En línea]. Consultado el 20 de abril de 2015 en: http://oiiinternational.com/

Ospina, C. A., y Runge, A. K. (2016). Degeneración, regeneración y raza: el proyecto moderno en Antioquia, 1903-1930. Anuario Colombiano de Historia Social y de la Cultura 43(2), 215-241.

Pedraza, Z. (1997). El debate eugenésico: una visión de la modernidad en Colombia. Revista de Antropología y Arqueología 9 (1-2), 115-159.

Prada, N. (2012). Diagnóstico de situación de personas intersexuales en la ciudad de Bogotá. Bogotá: Secretaría de Planeación, Alcaldía Mayor de Bogotá.

Preciado, B. (2003). Multitudes. Reveu politique, artistique, philosophique 12, [En línea]. Consultado el 27 de abril de 2017 en: http://www.multitudes.net/Multitudes-queer/.

Rubin, D. A. (2012). "An unnamed blank that craved a name": A genealogy of intersex as gender. Sings 37 (4), 883-908.

Runge, A. K. yMuñóz, D. A. (2005). El evolucionismo social, los problemas de la raza y la educación en Colombia, primera mitad del siglo XX: El cuerpo en las estrategias eugenésicas de línea dura y de línea blanda. Revista Iberoamericana de Educación (39), 127-168.

Sánchez, R. F. (1943). Se organiza la Sociedad de Obtetricia de Colombia. Revista Colombiana de Pediatría y Puericultura 2(5), 230.

Secord, J. A. (2004). Knowledge in Transit. ISIS (95), 654-672.

Sociedad de Obstetricia (1943) Heraldo Médico, Bogotá abril, p. 12.

Sterling, A.F. (2000). Cuerpos sexuados. [Traducción al español: García Letal, A.] (2006) Barcelona: Melusina. 
Tovar, J. V. (2016). Eugenesia en Colombia. Un problema de justicia social. Revista Colombiana de Bioética 11(1), 35-53.

Velásquez, C. et al. (2007). Principios y derechos involucrados en el análisis jurídico de los estados intersexuales en pacientes menores de edad en Colombia. -El caso del hermafroditismo-. Estudios de Derecho (64)144, 219-133.

Villegas, Á. A. (2005). Raza y nación en el pensamiento de Luis López de Mesa: Colombia, 1920-1940. Estudios políticos (26), 209-232. 